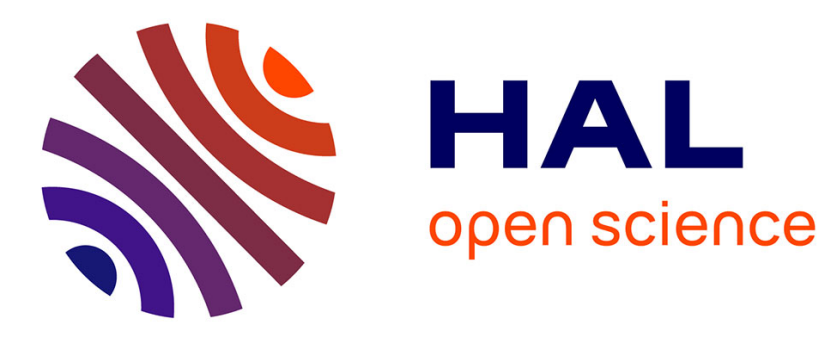

\title{
Three-dimensional effects in hovering flapping flight
} Thierry Jardin, Alain Farcy, Laurent David

\section{To cite this version:}

Thierry Jardin, Alain Farcy, Laurent David. Three-dimensional effects in hovering flapping flight. Journal of Fluid Mechanics, 2012, vol. 702, pp. 102-125. 10.1017/jfm.2012.163 . hal-01131936

\section{HAL Id: hal-01131936 https://hal.science/hal-01131936}

Submitted on 16 Mar 2015

HAL is a multi-disciplinary open access archive for the deposit and dissemination of scientific research documents, whether they are published or not. The documents may come from teaching and research institutions in France or abroad, or from public or private research centers.
L'archive ouverte pluridisciplinaire HAL, est destinée au dépôt et à la diffusion de documents scientifiques de niveau recherche, publiés ou non, émanant des établissements d'enseignement et de recherche français ou étrangers, des laboratoires publics ou privés. 


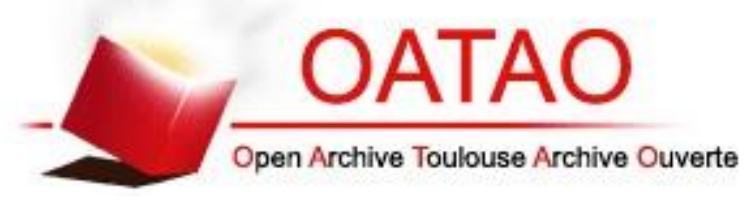

\section{Open Archive Toulouse Archive Ouverte (OATAO)}

OATAO is an open access repository that collects the work of Toulouse researchers and makes it freely available over the web where possible.

This is an author-deposited version published in: http://oatao.univ-toulouse.fr/ Eprints ID: 13652

To link to this article: DOI: $10.1017 / \mathrm{jfm} .2012 .163$

URL: http://dx.doi.org/10.1017/jfm.2012.163

To cite this version: Jardin, Thierry and Farcy, Alain and David, Laurent Three-dimensional effects in hovering flapping flight. (2012) Journal of Fluid Mechanics, vol. 702. pp. 102-125. ISSN 0022-1120 


\title{
Three-dimensional effects in hovering flapping flight
}

\author{
T. Jardin $\dagger$, A. Farcy and L. David \\ Institut Pprime, CNRS-Université de Poitiers-ENSMA, UPR3346, Département Fluides, \\ Thermiques, Combustion, SP2MI, Téléport 2, Bd Marie et Pierre Curie, \\ F-86962 Futuroscope Chasseneuil CEDEX, France
}

This paper aims at understanding the influence of three-dimensional effects in hovering flapping flight. Numerical simulations at a Reynolds number of 1000 are performed to compare two types of flapping kinematics whose plunging phase is characterized by either a rectilinear translation or a revolving motion. In this way, we are able to isolate the three-dimensional effects induced by the free end condition from that induced by the spanwise incident velocity gradient (and the associated implicit Coriolis and centrifugal effects). In the rectilinear translation case, the analysis of the wake and of the aerodynamic loads reveals that the wingspan can be compartmented into three distinct regions whether it is predominantly subjected to an unstable two-dimensional flow, a stable three-dimensional flow or both two-dimensional and three-dimensional effects. It is found that this partitioning exhibits common features for three different aspect ratios of the wing. In conjunction with the previous results of Ringuette, Milano \& Gharib (J. Fluid Mech., vol. 581, 2007, pp. 453-468), this suggests that the influence of the tip vortex over the wingspan is driven by a characteristic length scale. In addition, this length scale matches the position of the connecting point between leading and tip vortices observed in the revolving case, providing insight into the connecting process. In both translating and revolving cases, leading edge vortex attachment and strong spanwise velocities are found to be strongly correlated phenomena. Spanwise velocities (that mostly confine at the periphery of the vortices), together with downward velocities, do not only affect the leading edge vortex but also act as an inhibitor for the trailing edge vortex growth. As a consequence, crosswake interactions between leading and trailing edge vortices are locally limited, hence contributing to flow stabilization.

Key words: low-Reynolds-number flows, vortex interactions, vortex shedding

\section{Introduction}

Recently, flapping wings appeared as a possible alternative solution to the conventional fixed and rotary wing concepts for micro-air vehicles applications. The amount of lift generated by a flapping wing is essentially driven by four mechanisms;

$\dagger$ Present address: Université de Toulouse-ISAE, 10 av. Edouard Belin, 31055 Toulouse CEDEX 4, France. Email address for correspondence: thierry.jardin@isae.fr 
namely the leading edge vortex suction, the wake capture, the Kramer and added mass effects (Dickinson, Lehmann \& Sane 1999; Sane 2003; Shyy et al. 2010). The Kramer and added mass effects can directly be derived from the wing kinematics whereas the leading edge vortex formation and wake capture are intrinsically highly unsteady phenomena. The coupling between each of these mechanisms induces high sensitivity of the aerodynamic loads to the flapping kinematics. In this context, numerous studies focused on extracting two-dimensional relevant flapping parameters which could lead to enhanced aerodynamic performance (e.g. Milano \& Gharib 2005; Bos et al. 2008; Jardin, David \& Farcy 2009), sometimes to the detriment of understanding the fundamental physics which lie behind the complex three-dimensional unsteady flow.

One of the major issues that motivates current research on flapping wings aerodynamics concerns the stable attachment of the leading edge vortex observed on revolving wings. As opposed to two-dimensional configurations which predict a naturally unstable behaviour of the flow, a finite span wing revolving about its root is subjected to both a free end condition and spanwise incident velocity gradient (the translational velocity of the wing varies along the wingspan) that might be responsible for the attachment of the leading edge vortex through strong three-dimensional effects. Note that the spanwise incident velocity gradient is implicitly associated with Coriolis and centrifugal effects involved in the non-inertial wing frame. Although several researches greatly contributed to its understanding (Lentink \& Dickinson 2009a,b), the essence of the stable leading edge vortex attachment still remains unclear. In particular, the analysis of the flow that develops around a revolving wing is delicate due to the mutual interaction of free end and spanwise incident velocity gradient effects.

In view of isolating the role of the free end condition and that of the spanwise incident velocity gradient, we investigate and compare the flow around two types of flapping kinematics whose plunging phase is characterized by either a rectilinear translation or a revolving motion. In a first step, the translating case sheds light on the influence of the wing tip vortex and its dependence on the aspect ratio. Although some researchers did focus on finite aspect ratio translating wings at low Reynolds numbers (Ringuette, Milano \& Gharib 2007; Taira \& Colonius 2009; Kim \& Gharib 2010), very few studies report results based on finite aspect ratio flapping wings undergoing rectilinear translation (Shyy et al. 2009) and virtually none of them provide details of the influence of the wing tip vortex on global flow dynamics and specific flapping wing mechanisms such as the wake capture phenomenon. In a second step, lessons learned from the translating case bring insight into three-dimensional mechanisms that occur in the vicinity of revolving wings.

The present work relies on direct numerical simulations of the flow over a flapping NACA0012 aerofoil at a Reynolds number of 1000. The focus is on hovering flight for which the flow unsteadiness dominates quasi-steady effects.

\section{Kinematic parameters}

In both translating and revolving cases, the coordinates are set such that the $x$ - and $z$-axes are collinear to the wing displacement and wingspan, respectively, the $y$-axis being in the vertical direction.

\subsection{Translating case}

Figure 1(a) shows the trajectory followed by the wing for the translating case. The time evolution of the wing angle of attack $\alpha$, displacement $\delta^{*}$ (the origin is set at the mean wing position), rotation speed $\Omega^{*}$ (the centre of rotation $c_{0}$ is located $1 / 4$ 

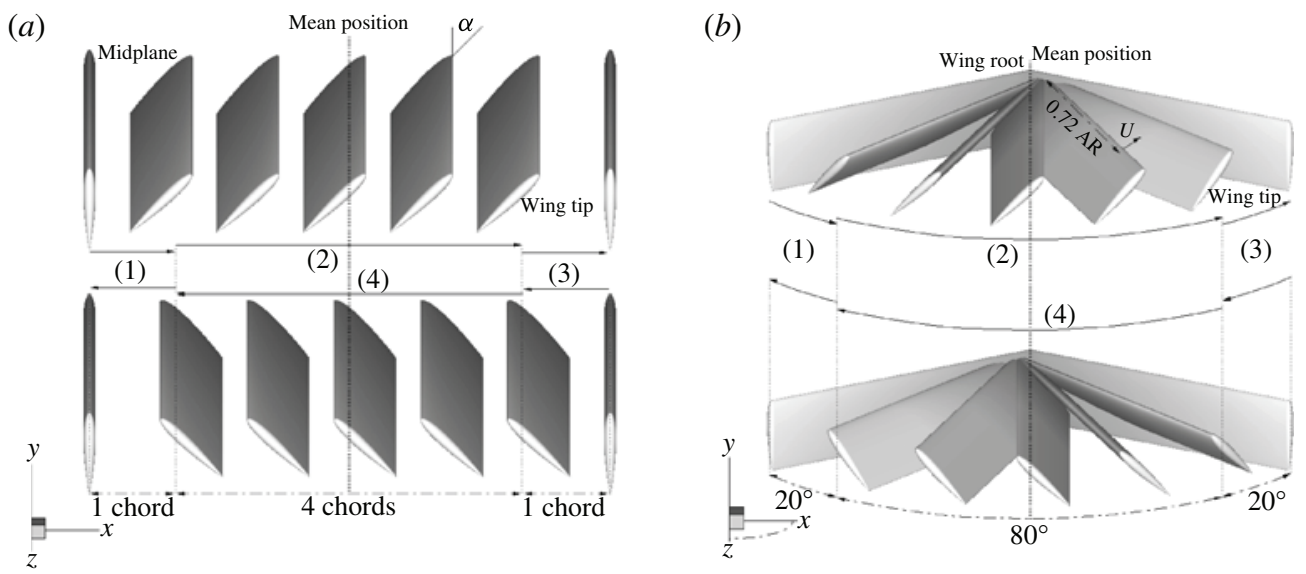

FIGURE 1. Illustration of the flapping motions: (a) translating case; (b) revolving case.
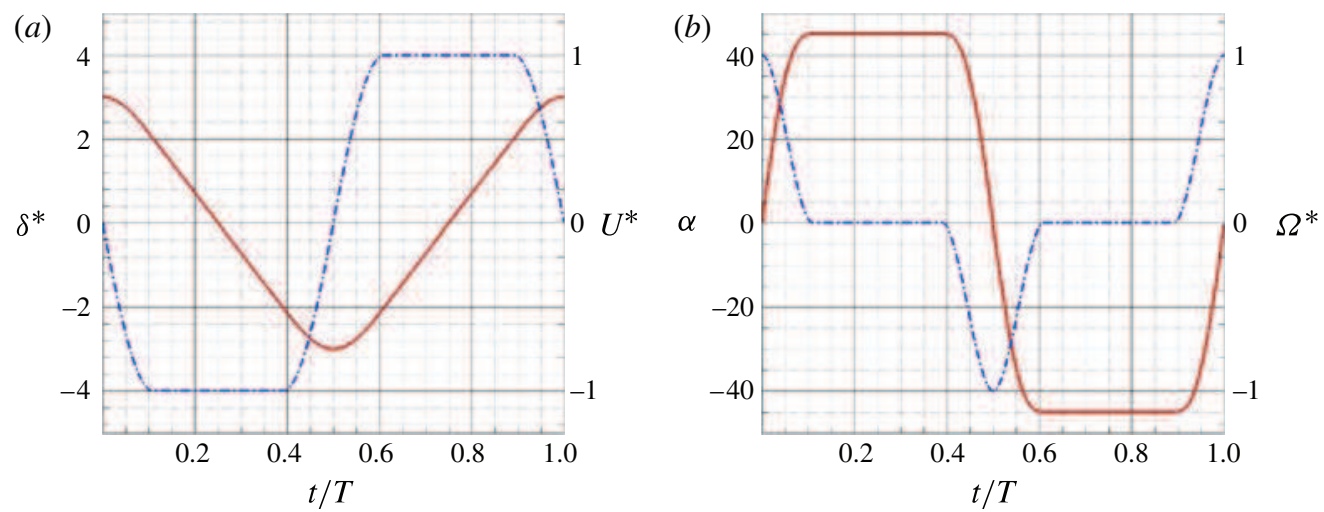

FIGURE 2. (Colour online available at journals.cambridge.org/flm) Evolution of the wing displacement $\delta^{*}$, speed $U^{*}$, angle of attack $\alpha$ and rotational speed $\Omega^{*}$ as a function of the flapping period fraction $t / T$.

chord away from the leading edge) and translation speed $U^{*}$ are depicted in figure 2 . Note that ${ }^{*}$ refers to non-dimensional values with respect to the chord $c$ and the maximum translation speed $U_{\max }$ of the wing. The flapping amplitude $A$ is set to six chords. Since we are concerned with the attachment of the leading edge vortex through three-dimensional effects, the amplitude is chosen such that it ensures leading edge vortex shedding in the absence of three-dimensional effects, i.e. $A>4$ chords (Dickinson \& Gotz 1993). The aerofoil undergoes a periodic (period $T$ ) motion defined by four distinct phases. The first (1) and third (3) phases are symmetrical with regard to the mean aerofoil position. They are characterized by a non-constant translation speed coupled with a rotating motion. The second (2) and fourth (4) phases extend four chords from the mean position. They are characterized by a constant translation speed and a fixed $45^{\circ}$ angle of attack. The aspect ratios $A R=2,4$ and 6 have been studied. Numerical simulations were conducted at a Reynolds number of 1000, based on the chord $c$ and the maximum translation speed $U_{\max }$ of the aerofoil. 


\subsection{Revolving case}

Figure 1(b) shows the trajectory followed by the wing for the revolving case. The flapping parameters defined for the translating case are projected on a spanwise section located at $R^{*}=0.72 A R$, where $R^{*}=0$ and $R^{*}=A R$ corresponds to the wing root and wing tip spanwise location, respectively. The time evolution of the wing angle of attack $\alpha$, displacement $\delta^{*}$, rotation speed $\Omega^{*}$ and translation speed $U^{*}$ in this specific spanwise section are therefore similar to those depicted in figure 2 . The translation speed variation along the wingspan $\partial U^{*}(t) / \partial R^{*}$ is equal to the revolving speed $\phi^{*}$ and is referred to as the spanwise incident velocity gradient. As evoked earlier, this specificity (i.e. non-zero $\left.\partial U^{*}(t) / \partial R^{*}\right)$ strongly contributes to the stability of the flow. In this case, six chords travelling at $R^{*}=0.72 A R$ corresponds to a $120^{\circ}$ flapping amplitude. Numerical simulations were conducted for the aspect ratio $A R=4$ at a Reynolds number of 1000 , based on the chord $c$ and the maximum translation speed $U_{\text {max }}$ reached in the $R^{*}=0.72 A R$ spanwise section.

\section{Investigation methods}

\subsection{Numerical methods}

The three-dimensional time-dependent Navier-Stokes equations were directly solved using a finite volume method. The aerofoil velocity in still air being of the order of $1 \mathrm{~m} \mathrm{~s}^{-1}$ (for a $1 \mathrm{~cm}$ chord), the following formulation was used, assuming incompressible flow:

$$
\begin{gathered}
\frac{\partial u_{i}}{\partial x_{i}}=0 \\
\frac{\partial u_{i}}{\partial t}+\frac{\partial}{\partial x_{j}}\left(u_{i} u_{j}\right)=-\frac{1}{\rho} \frac{\partial p}{\partial x_{i}}+v \frac{\partial^{2}}{\partial x_{j}^{2}}\left(u_{i}\right) .
\end{gathered}
$$

The pressure-velocity coupling was obtained using an iterative Pressure Implicit Split-Operator scheme. The latter relies on the Euler implicit time stepping scheme associated with explicit correctors. The temporal accuracy hence lies between the first and the second order. The space discretization was achieved using second-order centred differences.

An O-type non-conformal structured grid with 2 million cells was used. The crosssectional grid displayed in figure 3 was divided into two parts: an inner mesh which ensures a low diffusivity and hence a coherent modelization of wing-wake interactions, and an outer mesh introduced to avoid dependencies of the results on the far field boundary conditions. The external diameter of the inner and outer parts was 8 and 30 chords, respectively. The cross-sectional grid was extruded according to Cartesian and cylindrical frames to model the wingspan and wing tip/root. Figures $3(b)$ and $3(c)$ display side views of the three-dimensional meshes used for the rectilinear translation and revolving motion cases, respectively.

The boundary conditions at the far field were implemented as pressure Dirichlet conditions, allowing the local flow to be inwards or outwards. The wing midplane of the rectilinear translation case was defined as a symmetric boundary condition. It is noteworthy to mention that this setup suppresses any possibility for asymmetric vortex shedding as observed by Taira \& Colonius (2009) for low-aspect-ratio translating wings. The aerofoil surface was modelled as a non-slip surface. Its speed was prescribed at each time step using a moving-mesh approach. One flapping period was discretized into approximately 1000 time steps. 
(a)

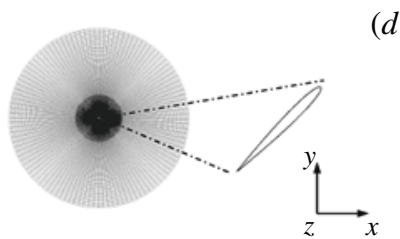

(b)

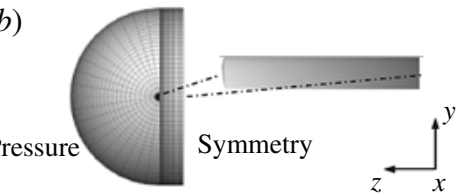

(c)

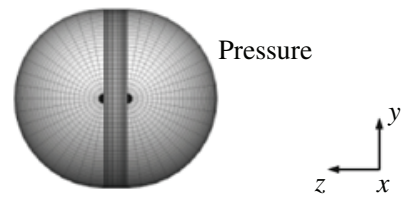

$(d)$

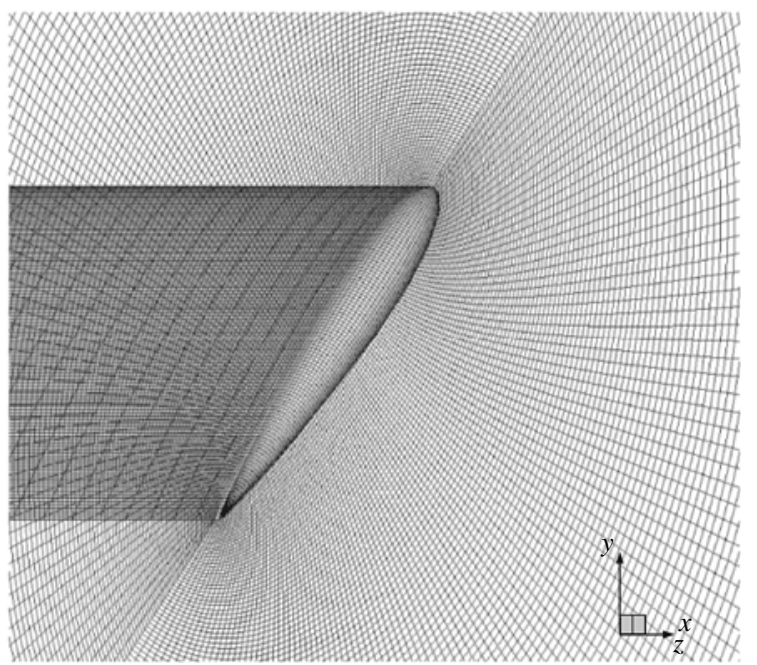

FIGURE 3. Computational domain: (a) cross-sectional mesh; (b) rectilinear translation side-view mesh; $(c)$ revolving motion side-view mesh; $(d)$ wing tip close-view mesh.

In what follows, results correspond to the 7 th period for which the flow is ensured to be periodical.

\subsection{Comparison with experimental data}

In order to provide a comparative basis, experiments were conducted for the rectilinear translation case shown in figure $1(a)$. A finite span transparent NACA0012 aerofoil was flapped in a water towing tank using two servo-controlled motors. Choosing water as the working fluid allows to operate at higher dimensions and lower speed, and thus gives the possibility to perform time-resolved spatially accurate particle image velocimetry (PIV) measurements. To reveal the three-dimensional flow structure that develops around the finite span aerofoil, i.e. to measure the three velocity components in a volume, we used a tomographic PIV three-dimensional $3 \mathrm{C}$ scanning technique. A Quantronix Darwin-Duo Nd:YLF laser scans a volume of $8 \mathrm{~cm}$ depth by means of an oscillating mirror. The volume is scanned within $25 \mathrm{~ms}(40 \mathrm{~Hz})$ which is small enough to consider the resulting particle mapping as instantaneous. It is discretized into 100 planes, involving the laser to operate at a frequency of $4 \mathrm{kHz}$ and leading to a spanwise resolution of approximately $0.8 \mathrm{~mm}$, to be compared with the $6 \mathrm{~cm}$ aerofoil chord. Particle images were captured by a high-speed synchronized Photron camera with a resolution of $1024 \times 784$ pixels. Cross-correlation between two successive volumes was then employed to obtain three-dimensional velocity flow fields. PIV processing was done using $32 \times 32 \times 8$ pixel interrogation windows. The complex and challenging process needed to realize such measurements is not described in detail here since it goes beyond the scope of the present paper. A more thorough description can be found in David et al. (2011).

Figure 4 compares the numerical and experimental non-dimensional out-of-plane vorticity contours and in-plane velocity vectors obtained in a spanwise plane at the beginning of the 7 th stroke. The plane is located 0.3 chords away from the wing tip where three-dimensional effects strongly influence the leading edge vortex behaviour. 
(a)

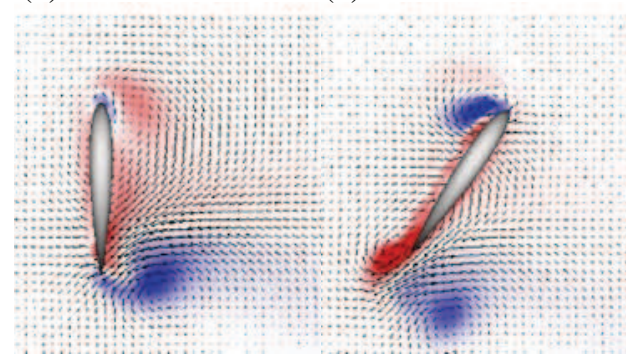

(e) $(f)$ (c)

$(d)$

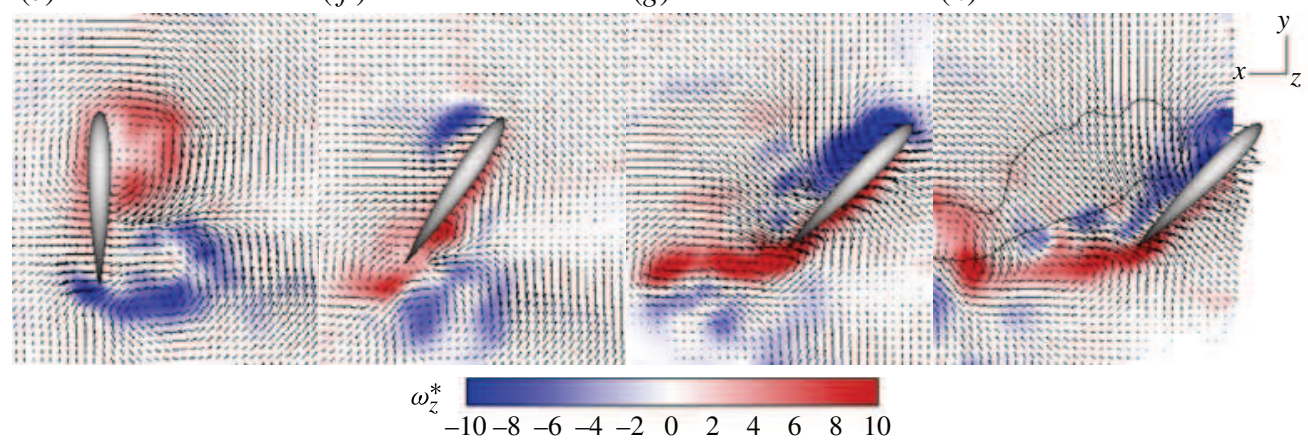

FIGURE 4. Comparison between $(a-d)$ numerical and $(e-h)$ experimental non-dimensional out-of-plane vorticity contours and in-plane velocity vectors obtained in a spanwise plane located 0.3 chords away from the wing tip at four instants $(a, e) 0 T / 20,(b, f) 1 T / 20$, $(c, g) 2 T / 20$ and $(d, h) 3 T / 20$ (from left to right). Spanwise (out-of-plane) velocity isolines $V_{z}^{*}=-0.25$ and $V_{z}^{*}=-0.20$ are depicted in frame $(d, h)$, respectively.

The plunging phase is here described by a rectilinear translating motion and an $A R=4$ wing is considered.

The sequence alternatively shows the wake structure arising from the previous halfstroke (figure $4 a, e$ ), its interaction with the wing (figure $4 b, f$ ) and the development of leading edge and starting vortices (figure $4 c, g$ and $d, h$ ). As will be detailed in $\S 4$, three-dimensional effects induced by the free end condition ensures the confinement of the leading edge vortex and attenuates wing-wake interactions. In this configuration, three-dimensional effects primarily appear as a broad inward $(-z$ direction) velocity region downstream the aerofoil. Despite discrepancies in characteristic levels, similar trends regarding the global behaviour of the main vortical structures and their interplay with three-dimensional effects are observed.

\subsection{Investigated quantities}

The vortical structures of the flow are depicted using the non-dimensional $\lambda_{2}^{*}$ criteria proposed by Jeong \& Hussain (1995).

The intensity of the vortices is evaluated using the non-dimensional circulation $\Gamma^{*}$ computed in a given vortex cross-section:

$$
\Gamma^{*}=\frac{1}{U_{\max } c} \iint_{S} \omega_{o p} \mathrm{~d} S
$$




$\begin{array}{lcccccc}\text { Blade element no. } & 1 & 2 & 3 & 4 & 5 & 6 \\ \text { Width relative to wingspan (\%) } & 28 & 22 & 17.5 & 13.5 & 10.5 & 8.5\end{array}$

TABLE 1. Width of the six blade elements used for local integration of drag and lift coefficients. Values are given as a percentage of the wingspan. Here element 1 and element 6 are respectively located at the wing midplane/root and wing tip.

where $S$ is the area contained within the $\lambda_{2}<-1$ region and $\omega_{o p}$ the out-of-plane vorticity.

In addition, the analysis is conducted on the basis of aerodynamic loads obtained locally on predefined spanwise elements of the wing (or blade elements, el.). The lift and drag coefficients are defined as

$$
C_{D, e l .}=\frac{2 F_{D, e l .}}{\rho A R_{e l .} c^{2} U_{\max }^{2}}, \quad C_{L, e l .}=\frac{2 F_{L, e l .}}{\rho A R_{e l .} c^{2} U_{\max }^{2}},
$$

where $F_{D, e l .}$ and $F_{L, e l}$. are the wing element's drag and lift forces, $c$ is the aerofoil chord and $A R_{e l}$. is the wing element's aspect ratio. The wingspan is discretized into six elements (table 1). For the revolving case, $U_{\max }$ depends on the wing element:

$$
U_{\max }(e l .)=\frac{1}{A R_{e l .} \times c} \int_{z_{e l .}^{-}}^{z_{e l .}^{+}} \phi_{\max } R \mathrm{~d} R
$$

where $z_{e l .}^{-}$and $z_{e l .}^{+}$are the lower and upper limits of element $e l$. along the wingspan and $R$ the spanwise position (recall that, for $A R=4, R^{*}=0$ and $R^{*}=4$ correspond to the wing root and tip section, respectively).

The contributions of the Kramer, rotational and translational added mass effects to the normal force $F_{N}$ experienced by the whole wingspan can simply be derived from the wing kinematics (Ellington 1984):

$$
\begin{gathered}
F_{N, \text { Kramer }}=\rho U \pi \Omega c^{2}\left(\frac{3}{4}-c_{0}\right) \\
F_{N, R A M}=\frac{1}{4} \rho \pi c^{2}\left[\frac{\partial \Omega}{\partial t} c\left(\frac{1}{2}-c_{0}\right)+\Omega U\right] \\
F_{N, T A M}=\frac{1}{4} \rho \pi c^{2} \frac{\partial U}{\partial t} .
\end{gathered}
$$

\section{Translating case}

\subsection{Wake topology}

Figure 5 shows equally spaced snapshots of the non-dimensional $\lambda_{2}^{*}$ isosurfaces obtained numerically during half of the 7 th flapping period. For sake of clearness, the flow fields are flipped with respect to figure 1 $(a)$. The flow region extending from the wing midplane to the wing tip is depicted for the aspect ratio $A R=4$.

In figure $5(a)$, the aerofoil is at its leftmost position, heading to interact with the wake generated during the previous half-stroke. The latter exhibits two pairs of dominantly spanwise (z-direction) counter-rotating vortices as well as a dominantly 
(a)

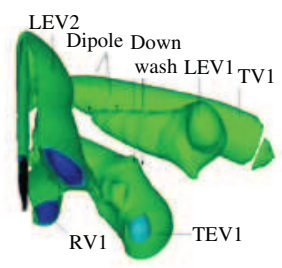

(b)

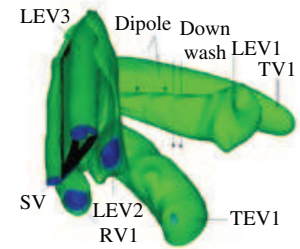

(c) $\operatorname{LEV}^{\mathrm{TV} 2}$

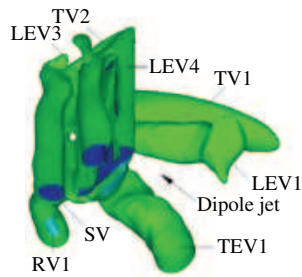

(d)

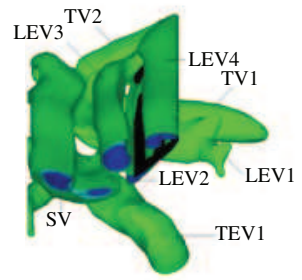

(e)

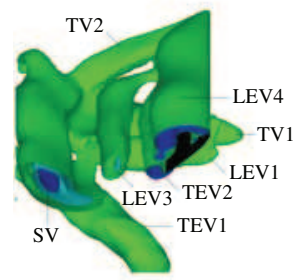

(f)

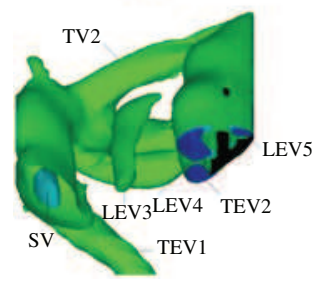

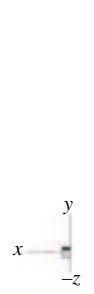

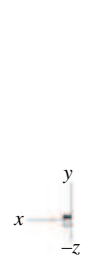

(h)
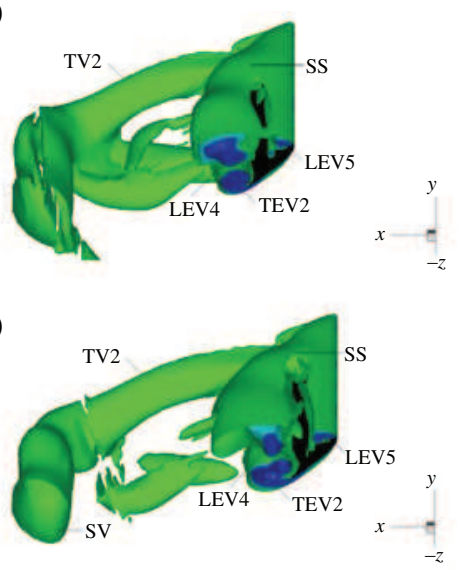

(i)

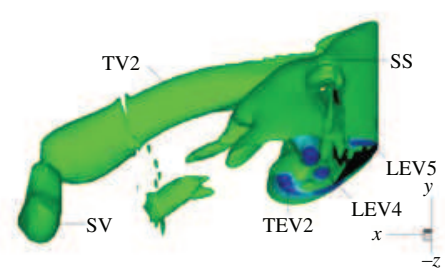

(j)

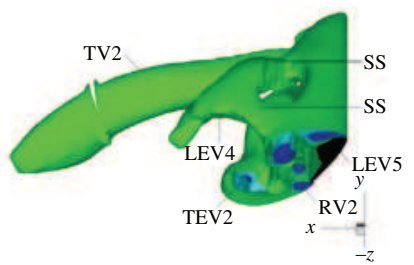

(k)

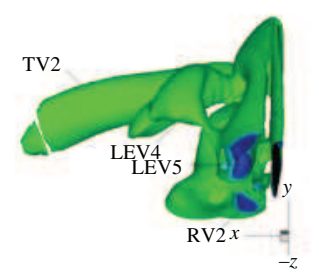

(l)

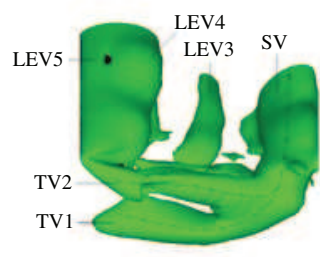

FIGURE 5. (Colour online) $\lambda_{2}^{*}$ iso-surfaces (green: -0.1 , light blue: -1 , blue: -2 ) obtained at $(a-k) t=n T / 20(n \in[0 ; 10])$ and $(l)$ flipped view at $t=5 T / 20$. Flow around an $A R=4$ flapping wing undergoing a rectilinear translation phase.

streamwise ( $x$-direction) tip vortex. The first pair consists of previously shed leading edge (LEV1) and trailing edge (TEV1) vortices. Their spanwise coherence is affected 
near the free end by the formation of a wing tip vortex (TV1). During the previous half-stroke, TV1 promoted the tilting of LEV1 and TEV1 in the streamwise direction. The mechanism leading to these streamwise structures (similar to SS on figure $5 g-j$ ) will be detailed later. Hence, near the free end, the flow arranges itself into a streamwise dipole from which originates a strong downward momentum flux (downwash). The second pair consists of a leading edge vortex (LEV2) generated during the later part of the previous half-stroke and a rotation vortex (RV1) induced at the trailing edge by the rotating motion. In contrast to the first vortex pair, this second vortex pair is predominantly two-dimensional since its late formation does not allow three-dimensional effects to affect its spanwise coherence.

As the aerofoil accelerates from left to right, it is noted from figure $5(b, c)$ that the interaction with the second vortex pair strengthens the formation of both leading edge (LEV3) and starting (SV) vortices. By locally enhancing the relative wing velocity, this interaction promotes the production of both leading and trailing edge shear vorticity and accelerates the natural shedding process. At this point, the newly formed structures LEV3 and SV are clearly two-dimensional. This characteristic is due to their quick shedding and to the two-dimensional behaviour of the wing-wake interaction. A consistent comparison can be done with strictly two-dimensional configurations (Jardin et al. 2009). Nevertheless, a substantial difference with two-dimensional cases arises from the rapid downward advection of LEV1 and TEV1 under the effects of the previously observed tip downwash. The influence of the fluid dipole jet arising from this first vortex pair, known to be beneficial for lift production (Birch \& Dickinson 2003), is hence less significant than observed for two-dimensional configurations.

The pure translating phase that follows is characterized by the concomitant formations of LEV4, TEV2 and TV2. It is noted from figure $5(d-i)$ that LEV4 evolves from a confined shape at the wing tip to a nearly two-dimensional shape at the wing midplane, revealing the influence of the free end through the formation of the tip vortex (TV2). Furthermore, it is shown that the TV2 is connected to both LEV4 and SV, leading to a complex vortex ring organization. Such flow pattern is closely linked to the advection of downward momentum responsible for insect flight (Wang 2004). A closer look at the wing tip region also reveals the connection between the vortex ring and TV1 (figure $5 l$ ). As such, although most vortices are labelled as independent entities to facilitate identification, one should note that the vortex organization TV2-SV-TV1 may be considered as a single entity in the sense of Helmholtz. The circulation of SV immediately after its shedding (at $t=2 T / 20$, before viscous dissipation tends to reduce its intensity) is similar to that of TV1 and TV2 just downstream the trailing edge, i.e. $\Gamma^{*}=0.9$. In addition, an interesting feature is the propagation of three-dimensional instabilities which modulate the leading edge vortex (LEV4) along the wingspan, as observed with bumps of $\lambda_{2}^{*}$ isosurfaces. This wavyness recalls the onset of hairpin vortex shedding observed in translating plate at low Reynolds numbers (Taira \& Colonius 2009).

Figure 5(g-i) show that the translating motion progressively leads to the shedding of LEV4, dividing the flow into three distinct regions:

(i) the near-tip region;

(ii) the near-midplane region;

(iii) the transition region.

The near-tip region is dominated by three-dimensional effects and the attachment of the leading edge vortex under the influence of the tip vortex. Since the leading edge vortex is here confined and closely attached to the aerofoil upper surface, this 
region is not very prone to severe wing-wake interactions. For instance, the leading edge vortex LEV2 generated during the previous half-stroke does not fundamentally alter the nascent leading edge vortex LEV3 in this region. It is shown from figure 5(c) that LEV3 remains attached to the aerofoil near the wing tip whereas LEV2 forces its shedding over most of the wingspan.

The near-midplane region is dominated by two-dimensional effects and the leading edge vortex shedding. The wing-wake interactions are hence very similar to that observed in strictly two-dimensional cases. For example, aside from the previously described interaction between the wing and LEV2, the flow is characterized by an increase of the aerofoil lower surface pressure due to the TEV1-LEV1 vortex dipoleinduced fluid jet.

The transition region is characterized by a streamwise structure (SS) that ensures continuity between the attached and separated parts of LEV4. This structure is promoted by the spanwise velocities associated with the formation of TV2. Therefore, the transition region is highly three-dimensional while being subjected to wing-wake interactions. It can be thought as a region of competition between three and twodimensional effects. It should be noted that the transition region grows towards the wing root as the aerofoil translates, according to the development of the tip vortex.

The continuity/discontinuity observed in the $\lambda_{2}^{*}$ isosurfaces suggests that the predominantly two-dimensional (near-midplane) region extends 2.6 chords away from the wing midplane. In the same way, the predominantly three-dimensional (near-tip) region extends 0.6 chords away from the wing tip. The remaining gap marks the transition region. These dimensions will be further quantified in $\S 4.3$.

From figure $5(i-k)$, the wing decelerates and rotates about its spanwise axis so as to ensure a proper orientation of the leading edge during the subsequent half stroke. Despite the fact that the near-midplane region is dominated by two-dimensional effects, it still exhibits three-dimensional instabilities. Figure $5(j)$ indicates that a second streamwise structure (SS) is formed in the vicinity of the wing root at the end of the stroke. At this instant, the flow is characterized by two vortex pairs as well as a tip vortex akin to that observed at the beginning of the stroke.

\subsection{Aerodynamic loads}

The evidence of two-dimensional, three-dimensional and transition regions is further quantified by monitoring the aerodynamic loads obtained locally on predefined wing elements. According to the spatial limits detailed earlier (table 1), wing elements 1-3 lie within the two-dimensional near-midplane region (although wing element 3 slightly overlaps the transition region), wing element 4 lies within the transition region, wing element 5 lies within both the transition and three-dimensional regions and wing element 6 lies exclusively within the three-dimensional near-tip region.

Figure 6 shows the time histories of the locally integrated drag and lift coefficients resulting from the wake topology described in the previous section. In addition, the contributions associated with the Kramer effects as well as the rotational and translational added mass effects are plotted in figure 7 in order to distinguish inertial forces from vortex-related forces.

At the beginning of the stroke, the loads experienced by wing elements 1,2 and 3 (near-midplane region) exhibit successive peaks and valleys reflecting the influence of severe wing-wake interactions. As described previously, these interactions are manifest in the rapid growth and shedding of LEV3 and the increase of the pressure on the lower surface of the aerofoil due to TEV1-LEV1 dipole jet. The first event is responsible for the peak and valley observed at $t=0.04 T$ and $t=0.1 T$, respectively. 

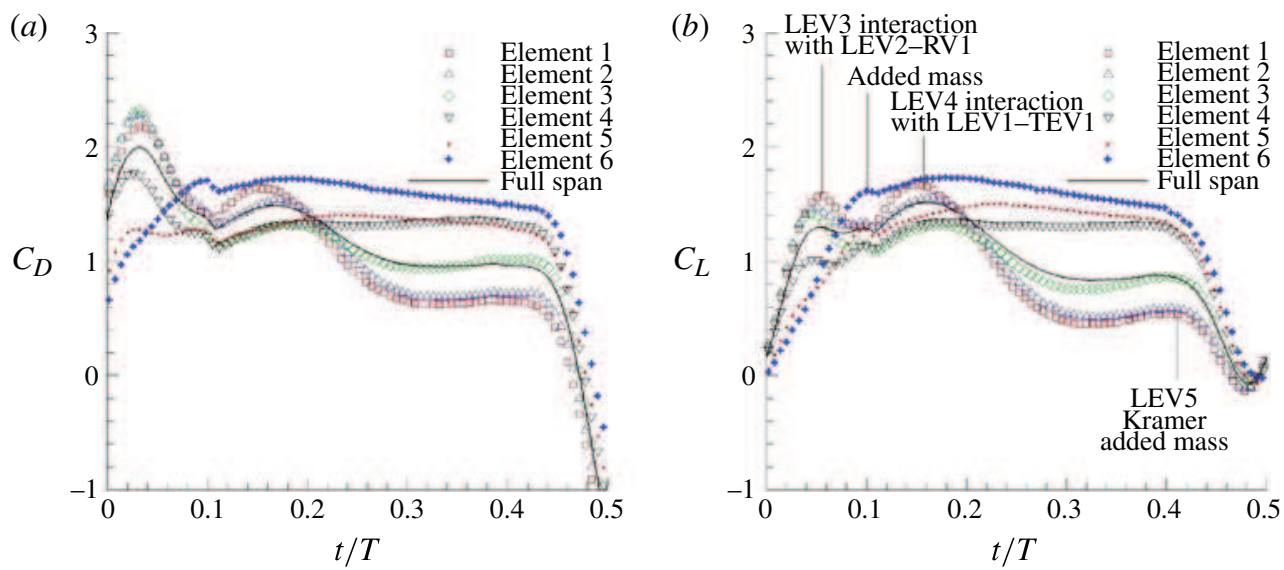

FIgURE 6. (Colour online) Time histories of the (a) drag and (b) lift coefficients experienced by each wing element of an $A R=4$ flapping wing undergoing a rectilinear translation phase.
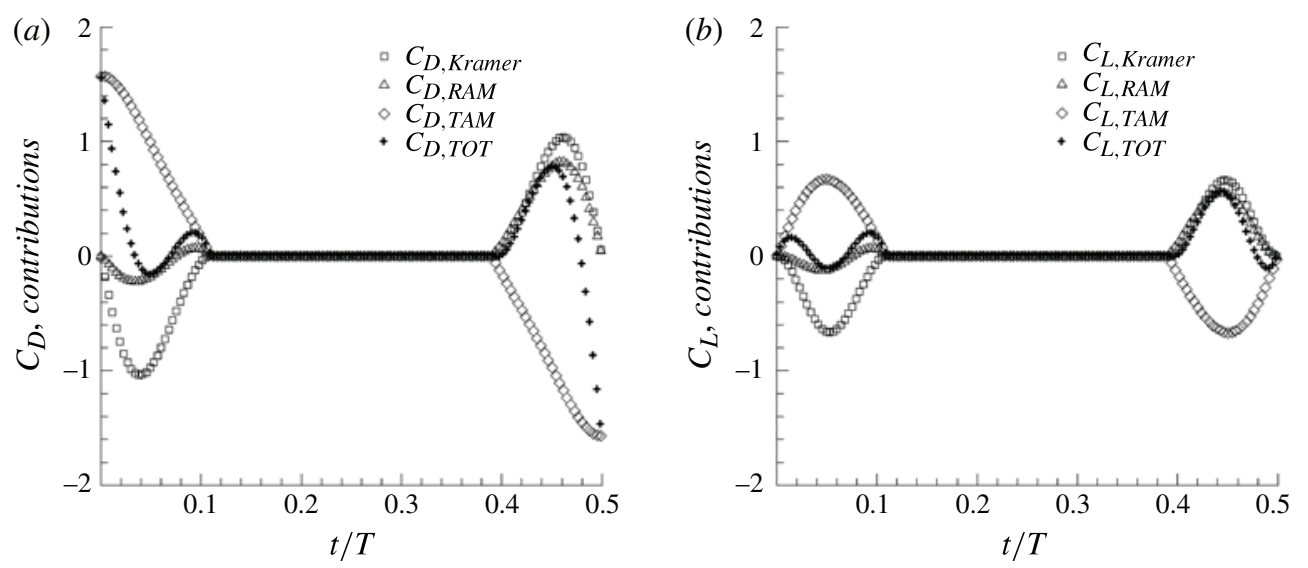

FIgURE 7. Time histories of the Kramer and added mass contributions to $(a)$ drag and $(b)$ lift coefficients experienced by an $A R=4$ flapping wing undergoing a rectilinear translation phase. Note that the range of the ordinate axis is different to that used in figure 6.

The second event occurs concomitantly with the growth of LEV4 and contributes to the peak observed at $t=0.16 T$. Afterwards, a sharp decrease leading to local minimum values of lift and drag at approximately $t=0.34 T$ is observed, indicating the shedding of the leading edge vortex LEV4 associated with the two-dimensional instability. This trend is slightly counteracted by the development of LEV5 as well as the Kramer and rotational added mass effects before drastically dropping under the influence of translational added mass effects.

On the other hand, the loads experienced by tip element 6 increase smoothly at the beginning of the stroke to reach strong values which persist until the aerofoil deceleration at $t=0.4 T$. Such behaviour clearly indicates the absence of significant wing-wake interactions and the continuous attachment of the leading edge vortex. In addition, as demonstrated previously by Ringuette et al. (2007) or Shyy et al. (2009), 


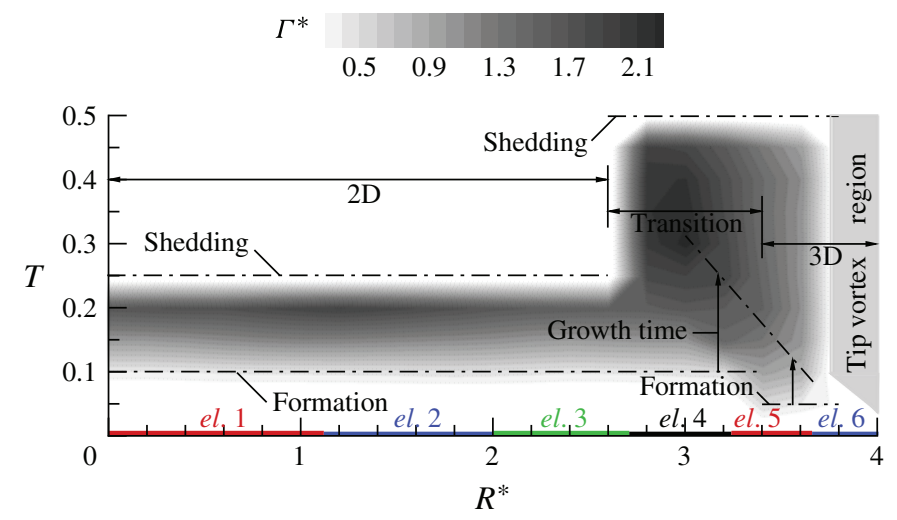

FIGURE 8. (Colour online) LEV4 non-dimensional circulation $\Gamma^{*}$ as a function of spanwise position $R^{*}$ and flapping period fraction $t / T$. Flow around an $A R=4$ flapping wing undergoing a rectilinear translation phase.

the tip vortex is primarily responsible for such high local values of lift and drag. The latter extends over $\sim 70 \%$ of element 6 and exhibits levels of circulation of the order of $\Gamma^{*}=0.9$ (evaluated just downstream the trailing edge). In contrast, the leading edge vortex circulation does not exceed $\Gamma^{*}=0.7$ (see figure 8 in the next section). It is noteworthy to mention that the small bump observed near $t=0.1 T$ on both drag and lift curves coincides with the end of the acceleration phase and accounts for added mass effects. Similarly, added mass effects are responsible for the sharp decrease that occurs after $t=0.4 T$.

Finally, the time histories of the drag and lift coefficients related to wing element 4 (transition region) are subjected to both sharp variation and quasi-steady phases, induced by wing-wake interactions and flow stabilization (leading edge vortex attachment), respectively.

Therefore, the signature of dominant vortical structures on aerodynamic loads helps in analysing in a more quantitative way the existence of three distinct flow regions. For the aspect ratio $A R=4$, the free end condition introduces substantial changes in the time history of total drag and lift coefficients in comparison to strictly two-dimensional flows (Jardin et al. 2009). In particular, the presence of a highly three-dimensional region near the wing tip is apparent through the attenuation of wing-wake interaction peaks and the increase in leading edge vortex induced forces.

\subsection{Leading edge vortex characterization}

A more detailed correlation between the flow structures and the force generation is achieved by evaluating the leading edge vortex intensity. Figure 8 displays the non-dimensional circulation $\Gamma^{*}$ of LEV4 obtained at different spanwise positions and instants. Spanwise positions $R^{*}=0$ and $R^{*}=A R=4$ denote the midplane and tip cross-sections, respectively. Times $t=0$ and $t=0.5 T$ correspond to the beginning and end of a half-stroke, respectively. The time-space diagram is discretized into 21 positions and 11 instants. White contours (i.e. zero values) are used before vortex formation and after vortex shedding so as to clearly point out the stable and unstable regions of the flow.

It is shown that the main leading edge vortex (LEV4) forms around $t=0.1 T$ in both near-midplane and transition regions. Here, the vortex formation is delayed with 
respect to the near-tip region where the leading edge vortex forms at about $t=0.05 T$. This delay is attributable to the preliminary formation and shedding of LEV3, which is a consequence of significant wing-wake interactions that occur in the near-midplane and transition regions.

In the near-midplane region (two-dimensional), LEV4 is then subjected to a constant growth and reaches a maximum intensity near $t=0.2 T$. This circulation threshold that eventually leads to the onset of vortex shedding around $t=0.25 T$ oscillates between $\Gamma^{*}=1.6$ and $\Gamma^{*}=1.9$. This behaviour is reminiscent of the three-dimensional instabilities that propagate along the near-midplane region, leading to slight spanwise variations in the development of LEV4.

The near-tip (three-dimensional) leading edge vortex evolution is fundamentally different. In this highly three-dimensional region, the flow evolves towards a stable state characterized by a plateau in the leading edge vortex intensity, as indicated by vertical isolines. The latter also suggest the increase of circulation from the wing tip towards the two-dimensional region. Such a feature is directly related to the increase of leading edge vortex growth time (time between vortex formation and the tilting of horizontal isolines into vertical isolines) with increasing distance from the tip. In particular, it is shown that the maximum circulation attained in the near-tip region is of the order of $\Gamma^{*}=1.5$ at $t=0.2 T$ and 0.6 chords away from the wing tip. Interestingly, this value does not exceed the circulation threshold that leads to vortex shedding in the near-midplane region, i.e. $\Gamma^{*}<1.6$. Therefore, flow stability can here simply be ensured by inhibiting local growth of the leading edge vortex.

In the transition region, the vortex growth persists beyond $t=0.2 T$. The circulation hence reaches strong values, up to $\Gamma^{*}=2.2$ at $t=0.3 T$ and 1 chord away from the wing tip. While the wing is still undergoing a constant speed translation after $t=0.3 T$, the circulation does not increase further though, suggesting that the tip vortex here again inhibits the vortex growth. This inhibition occurs lately in comparison to that observed in the tip region due to the time needed for tip vortex effects to propagate towards the two-dimensional region. As explained previously, this event is here again made evident through spanwise variation of the leading edge vortex intensity depicted by vertical isolines. However, the circulation levels are substantially higher than those attained within the unstable region, i.e. sufficient to provoke vortex shedding. As a consequence, an additional three-dimensional mechanism should be considered to explain the vortex attachment in the transition region.

It is well known that the vortex shedding process behind a circular cylinder is associated with both saturation and cross-wake interactions of upper and lower vortices (Williamson 1996). As such, inspecting the behaviour of the trailing edge vortex might bring insight into the physics that govern the leading edge vortex attachment in the transition region. To this avail, the non-dimensional $\lambda_{2}^{*}$ isosurfaces coloured by non-dimensional spanwise velocities ( $z$-direction) are depicted in figure 9 for two instants bounding the vortex shedding. In addition, spanwise cross-sections are displayed in figure 10. It has to be mentioned that spanwise velocities are here used as a marker of the intensity of the tip vortex. The latter further induces velocities in the $x$ - and $y$-directions that cannot be distinguished from two-dimensional-related $x$ - and $y$-velocities and that inevitably influence the behaviour of the leading edge and trailing edge vortices. In particular, downward (negative $y$-direction) velocities are expected to reduce the effective wing angle of attack, hence locally mitigating the production of spanwise vorticity.

Prior to vortex shedding (figures $9 a$ and $10 a-d$ ), the trailing edge vortex core (represented by the dashed line) demonstrates two distinct behaviours whether it is 
(a)

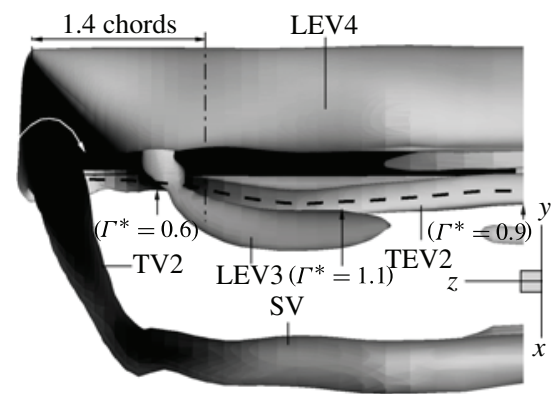

(b)

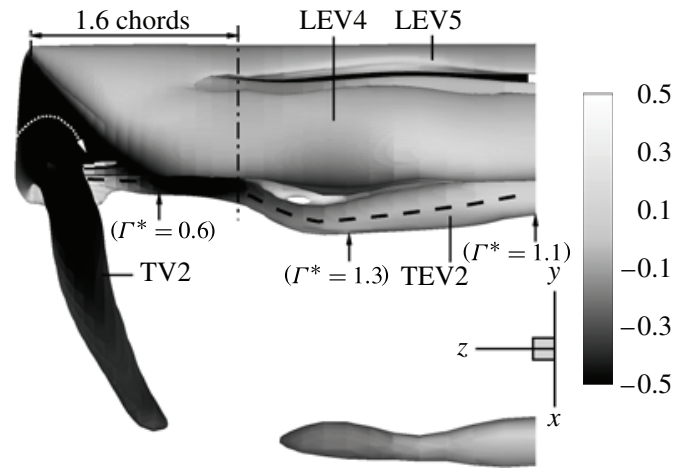

FIGURE 9. Non-dimensional $\lambda_{2}^{*}=-1$ isosurfaces coloured by non-dimensional spanwise velocities obtained at $(a) t=0.20 T$ and $(b) t=0.25 T$. Flow around an $A R=4$ flapping wing undergoing a rectilinear translation phase.

located near the tip or near the midplane. Near the wing tip, TEV2 is characterized by weak levels of circulation, of the order of $\Gamma^{*}=0.6$, and develops in the vicinity of the trailing edge. Strong spanwise and downward velocities arising from TV2, and to a minor extent by the streamwise ( $x$-direction) part of LEV3, are correlated with its confinement. Near the wing midplane, TEV2 is not affected by significant spanwise velocities and tends to move downstream the trailing edge, reaching circulation up to $\Gamma^{*}=1.1$. The frontier between these two behaviours is found to match the one that delimits the stable from the unstable parts of the flow, i.e. 1.4 chords away from the wing tip. These observations suggest that TV2 also acts as an inhibitor for the trailing edge vortex in such a way that the occurrence of cross-wake interactions between trailing and leading edge vortices is limited in the transition region, hence allowing a stable growth of LEV4.

The inspection of the flow topology after vortex shedding confirms the strong correlation between vortex confinement (both LEV4 and TEV2) and spanwise velocities. Figure $9(b)$ indicates that the trailing edge vortex circulation grows by 0.2 in the near-midplane region while it remains constant in the near-tip region. In addition, concomitantly to vortex shedding, it is shown that spanwise velocities propagate towards the midplane, extending the trailing edge vortex confinement up to 1.6 chords away from the wing tip. Spanwise cross-sections on figure $10(e-h)$ show that inward $(-z$ direction) velocities develop at the periphery of the vortices, essentially as a broad region downstream the leading edge vortex. At $t=0.25 T$, values up to $0.8 V_{0}$ can be reached in the near-tip region.

\subsection{Influence of the aspect ratio}

A straightforward question that arises from such results concerns the influence of the aspect ratio on unsteady flow dynamics and loads. Figure 11 displays the nondimensional $\lambda_{2}^{*}$ isosurfaces obtained for aspect ratios $A R=2, A R=4$ and $A R=6$ at $t=0.25 T$. The most prominent feature is that the flow encountered for $A R=2$ and $A R=4$ seems to be truncated replicas of that observed for $A R=6$. In other words, the absolute length of the three-dimensional and transition regions are independent of the aspect ratio. Increasing the aspect ratio is equivalent to extending the two-dimensional near-root region. The comparison between leading edge vortex intensities computed for each aspect ratio clarifies this observation. It can be seen from figure 12 that the 
(a)

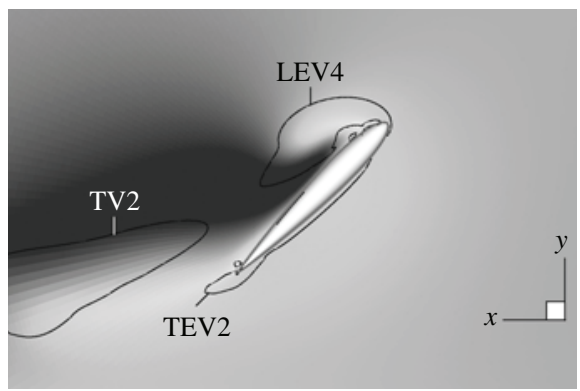

(b)

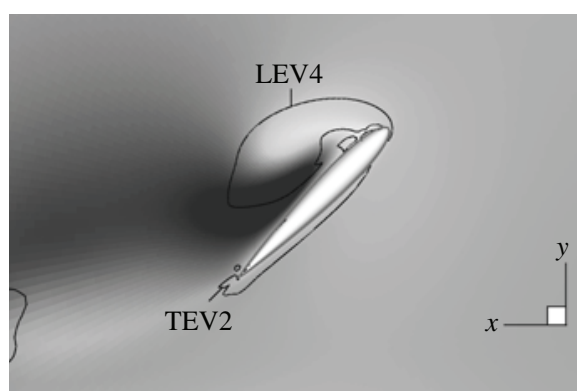

(c)

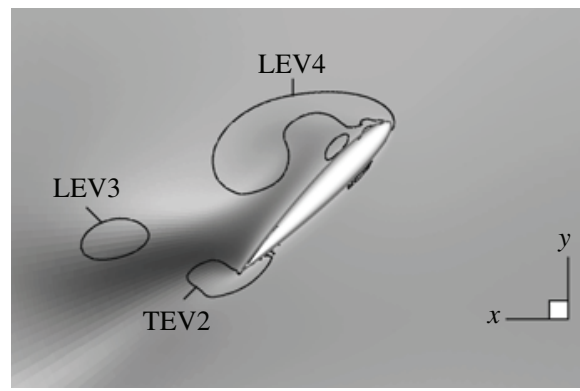

$(d)$

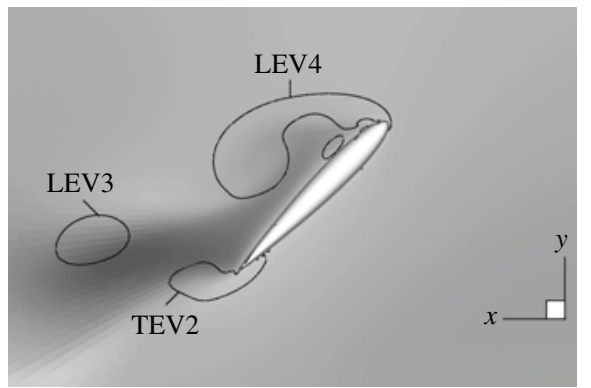

(e)

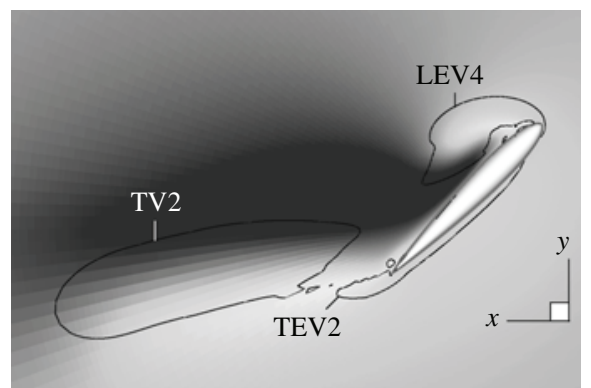

$(f)$

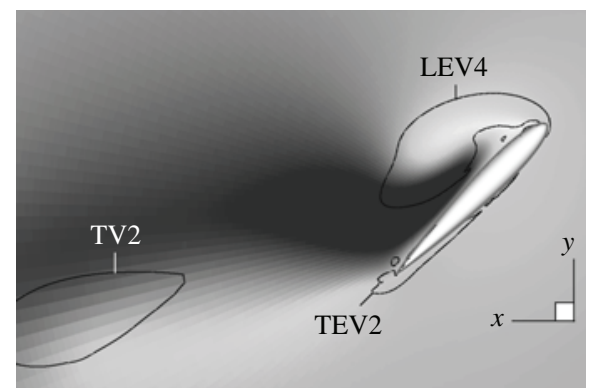

$(g)$

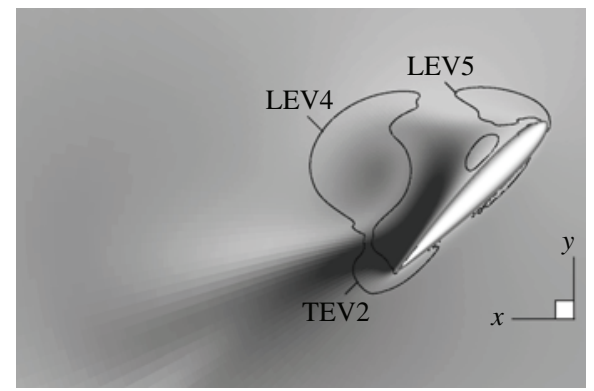

(h)

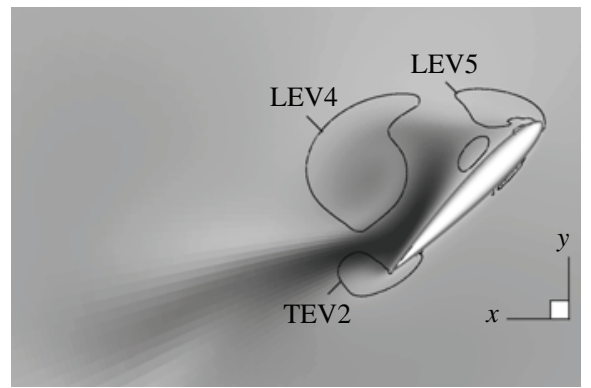

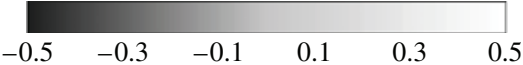

FIGURE 10. Non-dimensional spanwise velocity contours and $\lambda_{2}^{*}$ isolines obtained at $(a-d)$ $t=0.20 T$ and $(e-h) t=0.25 T$ in four spanwise cross-sections located $(a, e) 0.4,(b, f) 0.6$, $(c, g) 1.4$ and $(d, h) 1.6$ chords away from the wing tip. Flow around an $A R=4$ flapping wing undergoing a rectilinear translation phase.

circulation iso-lines evaluated for aspect ratios $A R=4$ and $A R=6$ are superimposed from the wing tip plane to the $A R=4$ midplane. In the $A R=6$ case, the isolines are 
(a)

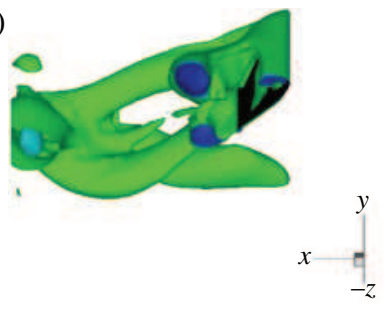

(b)

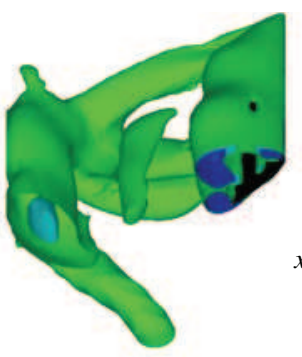

(c)

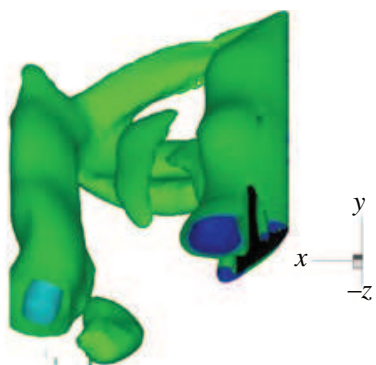

FIgURE 11. (Colour online) The $\lambda_{2}^{*}$ isosurfaces (green, -0.1 ; light blue, -1 ; blue, -2) obtained at $t=0.25 T$. Flow around (a) $A R=2,(b) A R=4$ and (c) $A R=6$ flapping wings undergoing a rectilinear translation phase.

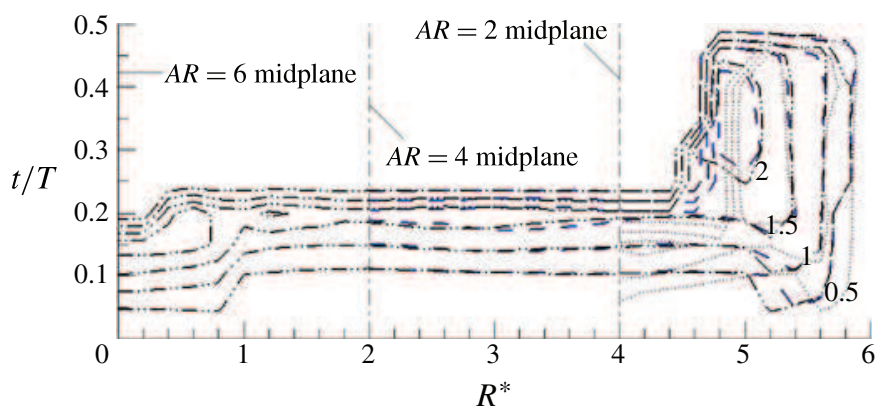

FIGURE 12. (Colour online) LEV4 non-dimensional circulation $\Gamma^{*}$ as a function of spanwise position $R^{*}$ and flapping period fraction $t / T$. Flow around (red dotted line) $A R=2$, (blue dashed line) $A R=4$ and (black dash-dotted line) $A R=6$ flapping wings undergoing a rectilinear translation phase. Note that offsets are applied to $R^{*}$ for the $A R=2$ and $A R=4$ cases to allow comparison.

extended beyond the $A R=4$ midplane and eventually undergo slight variations near the $A R=6$ midplane due to three-dimensional instabilities. The circulation isolines evaluated for aspect ratio $A R=2$ also show good agreement with the circulation isolines of $A R=4$ and $A R=6$ cases up to approximately 1 chord from the wing tip. However, in comparison to the $A R=4$ and $A R=6$ cases, the proximity of the symmetrical boundary condition (imposed at the midplane) and the free end condition slightly pushes the frontier between dominantly two-dimensional and threedimensional regions towards the wing tip. In addition, although it is not shown here for the sake of conciseness, tip vortices develop in a similar way for all three aspect ratios.

The overall comparison indicates that the relative influence of three-dimensional effects increase with decreasing aspect ratio. Hence, the flow that develops around low-aspect-ratio aerofoils is strongly three-dimensional and consequently not severely affected by wing-wake interactions. In this specific configuration (i.e. translating case with an amplitude fixed to $A=6$ chords), this results in enhanced mean aerodynamic lift and drag coefficients. Indeed, the gain associated with the leading edge vortex attachment encompasses the loss due to the attenuation of wing-wake interaction peaks, leading to $\overline{C_{L}}=0.99$ and $\overline{C_{D}}=1.16$ for $A R=2$ whereas $\overline{C_{L}}=0.96$ and 
$\overline{C_{D}}=1.14$ for $A R=4$ and $A R=6$. Such promotion may appear more significant for asymmetric flapping motions where flight is achieved by taking advantage of both lift and drag (Wang 2004).

\section{Revolving case}

\subsection{Wake topology}

An identical wake analysis is carried out for the revolving case described in figure $1(b)$. As opposed to the translating case, three-dimensional effects do not solely arise from the free end condition but from its coupling with the spanwise incident velocity gradient. The $\lambda_{2}^{*}$ isosurfaces are displayed in figure 13 for the 7 th flapping period.

In figure 13(a), the flow is considerably simplified in contrast to that observed for the rectilinear translation case. Three distinct vortices are identified, namely the TV1, the LEV1 and the RV1. RV1 is generated during the later part of the half-stroke from the rotational motion which does not involve spanwise incident velocity gradient. Thus, it exhibits a dominantly two-dimensional behaviour. On the other hand, TV1 and LEV1 reveal the existence of highly three-dimensional dynamics. As commonly observed in this range of Reynolds numbers (Ellington et al. 1996), LEV1 has a conical shape and connects to TV1 near the wing tip.

It can be seen from figure $13(b, c)$ that these three vortices connect into a ring-like structure which circumscribes the wing tip as the aerofoil accelerates from left to right. This pronounced wing-wake interaction promotes the development of newly formed TV2, LEV2 and SV. The latter are immediately highly three-dimensional due to the three-dimensional nature of the interacting wake vortices.

In figure $13(c)$, it is noted that the extreme part of SV is rapidly shed into the wake. Its connection with TV2 and LEV2 leads to the formation of a vortical annular ring which convects a strong downward momentum flux $(e-g)$, analogous to the production of lift. This flow organization is comparable to that identified by Sun \& Lan (2004) or Aono, Liang \& Liu (2008) for insect model wings. As a result from such downward momentum, TV2 and SV are advected beneath the trailing edge such that they do not significantly alter the subsequent half-stroke flow.

Meanwhile, LEV2 progressively develops on the upper surface of the wing, revealing a stable conical shape. Its spanwise cross-section grows from the wing root to the point where it connects to TV2. The connecting point is found to be approximately 3.4 chords away from the wing root (85\% of the wingspan) and is representative of a local instability. An interesting feature is the correspondence between the position of the LEV2-TV2 connecting point and the spanwise extent of the tip vortex dominated region (three-dimensional region) observed in the translating case. Such correspondence suggests that the connecting process results from the competition between tip vortex and spanwise incident velocity gradient effects. This point will be detailed in $\S 5.3$.

In addition, the formation of a trailing edge vortex is observed in the vicinity of the wing tip where LEV2 attains a significant size (figure 13l). Similarly to LEV2, TEV spanwise cross-section grows towards the wing tip and connects to a weaker secondary tip vortex. This connection is consistent with previous experimental results (Poelma, Dickson \& Dickinson 2006).

At the end of the stroke, the pronation phase induces the generation of a rotation vortex (RV2) at the trailing edge and the slight detachment of LEV2 from the leading edge. 
(a)

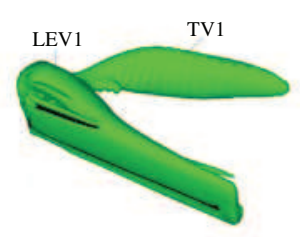

(b) Ring-like

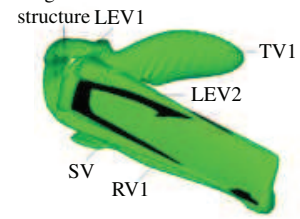

(c)

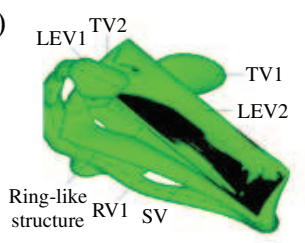

(d)

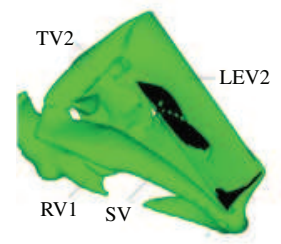

(e)

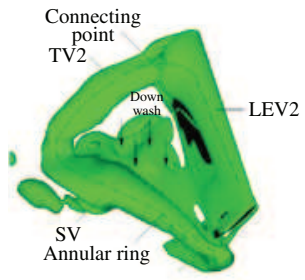

(f)

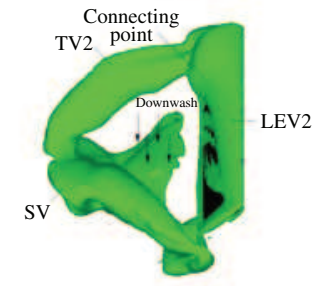

(g)

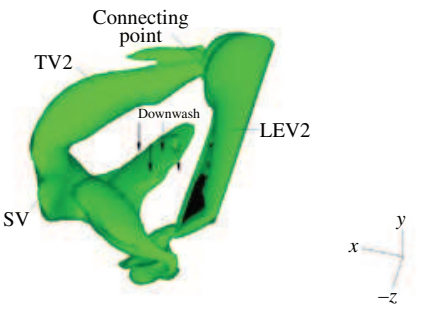

(h)

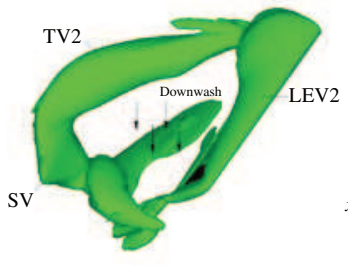

(i)

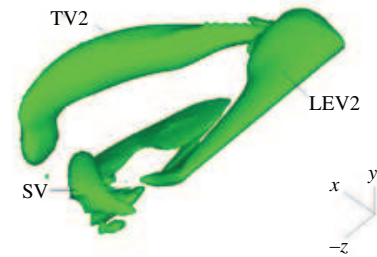

(j)



(k)

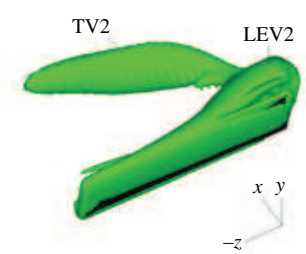

(l)

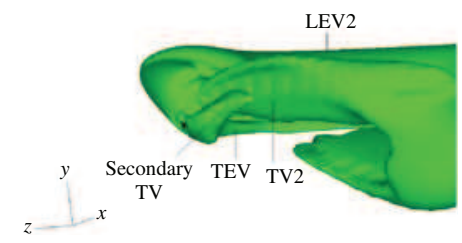

FIGURE 13. (Colour online) The $\lambda_{2}^{*}$ isosurfaces (green, -0.1 ; light blue, -1 ; blue, -2 ) obtained at $(a-k) t=n T / 20(n \in[0 ; 10])$ and $(l)$ rear view at $t=5 T / 20$. Flow around an $A R=4$ flapping wing undergoing a revolving motion. 

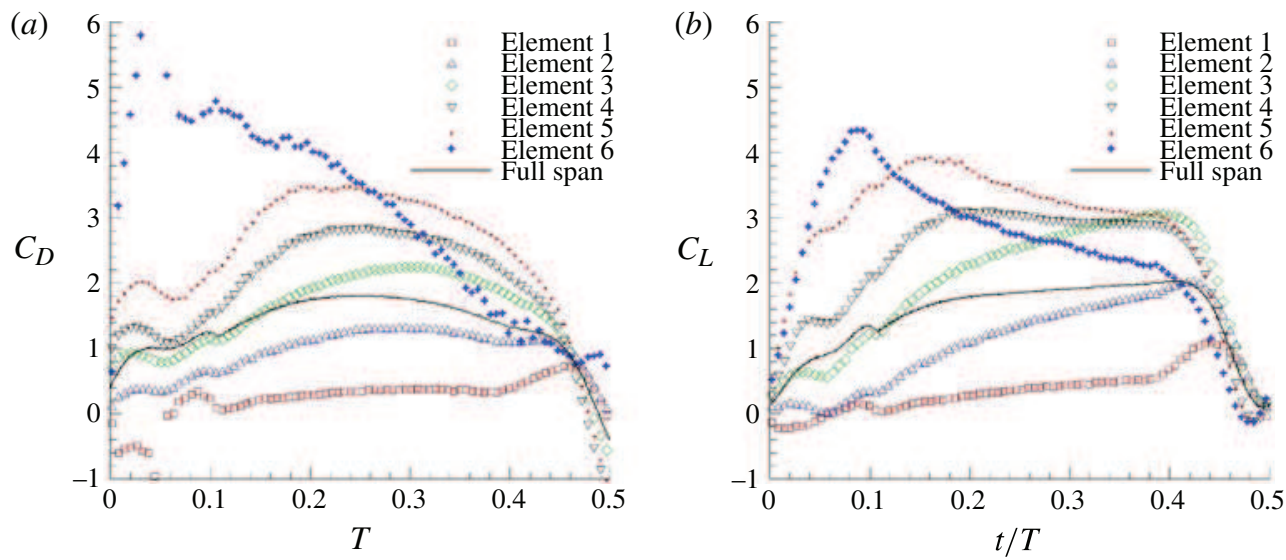

FIgURE 14. (Colour online) Time histories of the $(a)$ drag and $(b)$ lift coefficients experienced by each wing element of an $A R=4$ flapping wing undergoing a revolving motion.

\subsection{Aerodynamic loads}

The aerodynamic loads experienced by the wing emphasize the different regions of the flow. Figure 14 depicts the time history of locally integrated aerodynamic coefficients and highlights the role of wing-wake interactions, quasi-steady mechanisms associated with LEV2 attachment and LEV2-TV2 connecting process.

At stroke reversal, the trend associated with the wing-wake interactions is opposed to that highlighted for the rectilinear translation case. Here, near-tip wing element 6 is subjected to a strong drag which originates from the formation of a fluid jet oriented towards the lower surface of the wing. This fluid jet emanates from the presence of the ring-like structure identified in the vicinity of the wing tip and provokes a high-pressure region on the lower surface. On the other hand, near-root wing elements are not very prone to severe wing-wake interactions and thus provide weak drag.

This difference is further emphasized during the supination phase. Whereas wing elements 1-5 are subjected to slight variations typically linked to Kramer and added mass effects, the lift and drag experienced by element 6 sharply increase towards maximum values at $t=0.1 T$. This, again, is a consequence of the ring-like structure and its associated fluid jet which modifies the wing incident velocity and accentuates the leading edge separation, i.e. leading edge vortex formation. Yet, it is important to note that wing elements are subjected to different added mass effects with respect to the local wing acceleration (increasing from wing root to wing tip).

During the upstroke phase, the evolution of the lift coefficient reveals three distinct behaviours. Near the wing root, the quasi-linear and persistent increase of $C_{L}$ throughout the stroke suggests that the flow around elements 1,2 and 3 is not fully developed. In contrast, the flow around element 4 is associated with a more pronounced increase of lift, followed by a broad plateau that lasts until the pronation phase. This evolution indicates a rapid saturation of the leading edge vortex towards a quasi-steady state. Last, near the wing tip, elements 5 and 6 show a continuous decrease of $C_{L}$. In that region, the rapid growth of LEV2, promoted by wing-wake interactions, is counteracted by the development of the TV. This observation is detailed in the next section. 


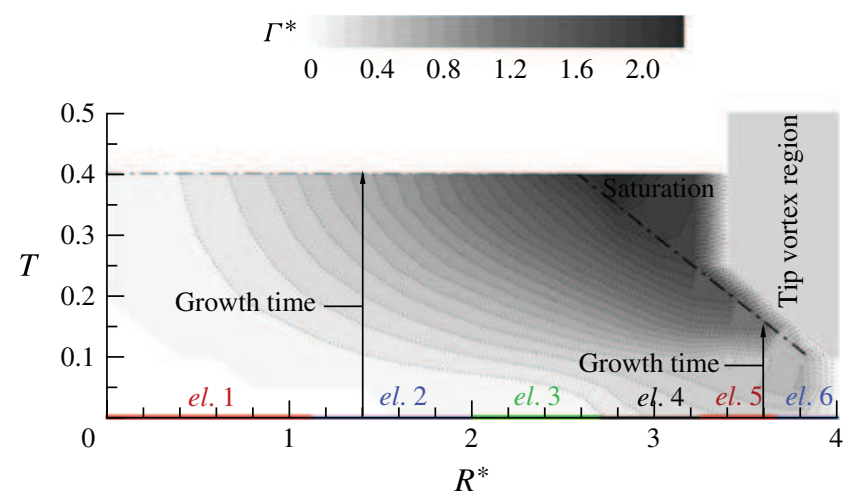

FIGURE 15. (Colour online) LEV2 non-dimensional circulation $\Gamma^{*}$ as a function of spanwise position $R^{*}$ and flapping period fraction $t / T$. Flow around an $A R=4$ flapping wing undergoing a revolving motion.

\subsection{Leading edge vortex characterization}

As previously achieved in $\S 4.3$, the correlation between flow topology and aerodynamic loads is revealed by the time-space evolution of LEV2 circulation computed on the interval $[0 ; 0.4 T]$ (figure 15), i.e. before wing deceleration and rotation. The evaluation of the circulation after $t=0.4 T$ is ambiguous due to the overlapping of $\lambda_{2}$ isolines, which results from the proximity of leading and trailing edge vortices and from solid-body rotation induced by the rotating wing.

In contrast to the translating case, the nascent leading edge vortex at $t=0$ is not subjected to rapid shedding under the influence of wing-wake interactions. Rather, the interaction of the wing with the ring-like structure depicted on figure 13(a) advances LEV2 formation such that the latter exhibits circulation exceeding 0.2 along the last quarter of the wingspan at the very beginning of the half stroke.

Afterwards, LEV2 develops according to the distance travelled by the wing. As such, from $R^{*}=0$ to $R^{*} \approx 2.8$, the concentric ellipses scheme depicted by the circulation isolines reveals the spanwise variation of the vortex growth, or LEV2 conical shape, associated with the variation of the local distance $\delta$ travelled by each wing crosssection. At this point, it is important to note that elliptic isolines roughly evolve into regularly spaced straight isolines if the circulation is plotted as a function of $\delta^{*}$ rather than $t / T$. In other words, the circulation grows linearly with the distance travelled by the wing, such that $\Gamma^{*}=C \delta^{*}$. Nevertheless, non-strictly parallel isolines reveal that coefficient $C$ varies along the wingspan. This is due to the fact that the budget between vorticity production at the leading edge and vorticity dissipation/transport is not constant along the wingspan, as suggested by the experimental studies of Poelma et al. (2006) and Kim \& Gharib (2010). Beyond this region (for $R^{*}>2.8$ ), LEV2 saturates or connects to TV2.

Near the free end, figure 15 indicates that TV2 propagates from the wing tip towards the wing root. This event is coincident with the drastic force decrease experienced by wing elements 5 and 6 . In this region, TV2 connects to LEV2, turning spanwise vorticity into streamwise vorticity.

Similarly, it is shown that LEV2 saturation (denoted by vertical isolines) coincides with the constant force experienced by wing element 4. Interestingly, the saturation process and the propagation of tip effects appear to be correlated phenomena, as was previously observed for the translating case. As such, saturation occurs lately 1.2 
(a)



$(c)$

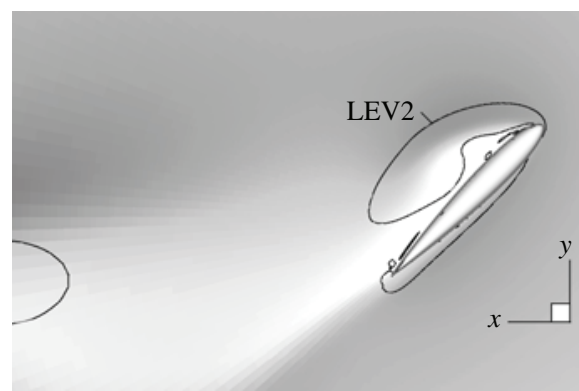

$(e)$

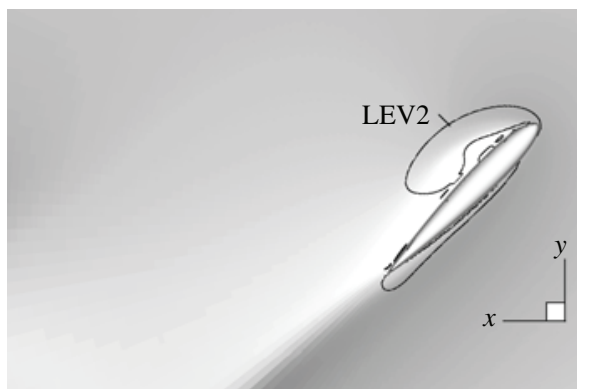

(b)

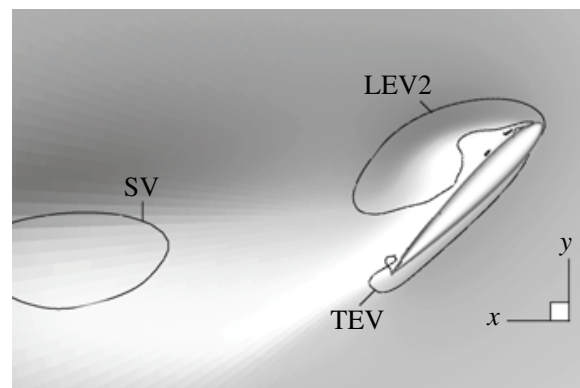

$(d)$

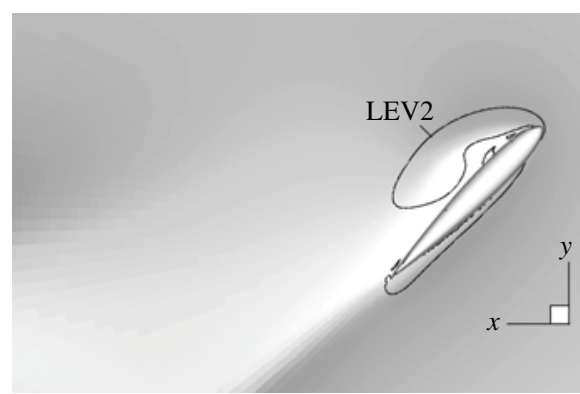

$(f)$

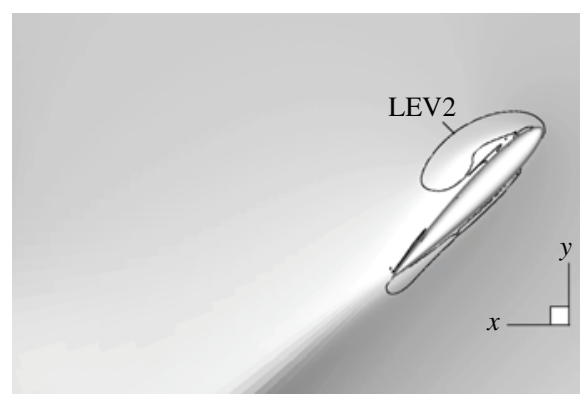

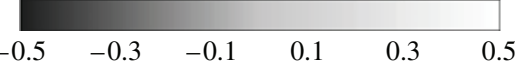

FIGURE 16. Non-dimensional spanwise velocity contours and $\lambda_{2}^{*}$ isolines obtained at $t=0.25 T$ in six spanwise cross-sections located $(a) 0.6,(b) 0.8,(c) 1.0,(d) 1.2,(e) 1.4$ and $(f) 1.6$ chords away from the wing tip. Flow around an $A R=4$ flapping wing undergoing a revolving motion.

chords away from the wing tip due to the time needed for tip effects to propagate towards the wing root. Hence, maximum circulations $\left(\Gamma^{*}=2.3\right)$ are attained 1.2 chords away from the wing tip at $t=0.4 T$.

In order to shed light on the physics that govern the behaviour of the leading edge vortex, we examine the non-dimensional $\lambda_{2}^{*}$ isolines superimposed to non-dimensional spanwise velocity contours in six cross-sections at $t=0.25 T$.

Figure 16 demonstrates that the continuous attachment of the leading edge vortex in the stable part of the flow (from the wing root to approximately 0.6 chords away form the wing tip) is associated with outward velocities that confine to the aerofoil upper surface at the periphery of LEV2, showing consistency with the experimental results of Kim \& Gharib (2010). Levels evolve from approximately $0.25 V_{0}$ at the wing root to $V_{0}$ near the connecting point. It is remarkable that the location of such 
outward velocities, induced by the revolving motion, coincides with the region of strong inward velocities, induced by the free end condition, observed in the rectilinear translation case (figure 10). In addition, it is shown that the position (along the wingspan) of the connecting point between LEV2 and TV2 matches the limit of the three-dimensional region observed in the translating case. Therefore, the physics that lead to the connection between LEV2 and TV2 can be interpreted as a four-stage process.

First, following the idea that outward velocities are responsible for leading edge vortex attachment on revolving wings (Ellington et al. 1996), inward velocities counteract outward velocities near the wing tip and promote local instability of the flow. Thus, LEV2 detaches from the leading edge near the wing tip but remains attached near the root. Akin to the formation of the SS structure in the translating case, this leads to the tilting of spanwise vorticity into streamwise vorticity. The resulting streamwise part of LEV2 and TV2 being co-rotating vortices, they connect into a single entity.

In comparison to the translating case, the tip vortex is thus moved up towards the leading edge such that continuity between outward velocities induced by both revolving motion and free end effects is ensured.

From figure 16, it is further noticeable that no significant trailing edge vortex is observed in the stable flow region. Similarly to the translating case, it is believed that the absence of strong cross-wake interactions between leading edge and trailing edge vortices contributes to the stabilization of the flow and allows LEV2 circulation to reach values much greater than the one obtained in unstable flow regions. Here again, spanwise cross-sections put into evidence the correlation between trailing edge vortex inhibition and spanwise velocities.

\section{Conclusion and discussion}

Interest in flapping wings aerodynamics has been increasing rapidly due to its possible application to micro-air vehicles. One of the main issues that motivates current research concerns three-dimensional effects arising from both the free end condition and spanwise incident velocity gradient.

We conducted numerical simulations to assess the influence of three-dimensional effects on the leading edge vortex behaviour and their consequences on wing-wake interaction mechanisms.

In view of isolating the three-dimensional effects induced by the free end condition from that induced by the spanwise incident velocity gradient, we first investigated the wake generated by a flapping wing whose translation phase was rectilinear. The analysis revealed that the flow can be compartmented into three distinct regions whether it is dominated by two-dimensional effects (pronounced wing-wake interactions, leading edge vortex shedding), three-dimensional effects (weak wing-wake interactions, leading edge vortex attachment) or by the propagation of three-dimensional effects towards two-dimensional effects (transition region).

In the three-dimensional region, the leading edge vortex growth is inhibited by tip effects. Its circulation saturates at levels inferior to those reached within the unstable region, consistent with its stable attachment. On the other hand, the leading edge vortex in the transition region is characterized by both stable attachment and high levels of circulation, superior to those reached within the unstable region. Its continuous attachment is here promoted by the trailing edge vortex inhibition (due to tip effects) and, as a consequence, by the attenuation of cross-wake interactions (between leading and trailing edge vortices). Such interactions are known to be associated with vortex shedding behind a circular cylinder (Williamson 1996). 
The dimensions of the stable and transition regions were found to be independent of the aspect ratio. In conjunction with the previous results of Ringuette et al. (2007), who reported similar dimensions for a translating normal flat plate, this suggests that the influence of the tip vortex over the wingspan is driven by a characteristic length scale. The wing portion exhibiting continuous attachment of the leading edge vortex under the influence of tip effects extends 1.4 chords away from the wing tip. Therefore, it is shown that stable flow cannot be obtained on a flapping wing undergoing rectilinear translation phase for aspect ratio exceeding 1.4. This clarifies the hypothesis of Birch \& Dickinson (2001) who suggested that tip effects alone could be responsible for leading edge vortex attachment at low Reynolds numbers.

We then focused on a flapping wing undergoing a revolving motion. As expected (Ellington et al. 1996), the leading edge vortex exhibits a conical shape and connects to the tip vortex near the free end.

It is shown that the position of the connecting point matches the limit of the three-dimensional region observed in the translating case ( 0.6 chord away from the wing tip). In addition, the region of strong inward velocities induced by the tip vortex (in the translating case) corresponds to that of strong outward velocities induced by the spanwise incident velocity gradient (in the revolving case). Thus, inward velocities counteract outward velocities, giving rise to a local instability. As such, the leading edge vortex detaches near the wing tip but remains attached along $85 \%$ of the wingspan. This provokes the tilting of the leading edge vortex into the streamwise direction. The leading edge and tip vortices being co-rotating, they connect into a single entity.

Beyond the connecting point (towards the wing tip), the flow is unstable and significantly affected by wing-wake interactions. Before the connecting point (towards the wing root), the flow is stable and weakly affected by wing-wake interactions. In this stable region, various authors (Maxworthy 1979; Ellington et al. 1996; Lentink \& Dickinson 2009b) suggested that spanwise flow balances vorticity production at the leading edge, hence allowing the continuous attachment of the leading edge vortex. Nevertheless, the leading edge vortex can reach high levels of circulation, similar to those attained within the transition region of the translating case and superior to those attained within unstable regions. Here, again, we suggest that the trailing edge vortex is strongly inhibited, which contributes to the attenuation of cross-wake interactions and to flow stabilization. Such inhibition is associated with strong outward velocities that mostly confine to the upper surface of the aerofoil and eventually extend to a broad region downstream the leading edge vortex.

As a conclusion, analogies between translating and revolving wings brought further insight into the mechanisms that govern the leading edge vortex behaviour. The comprehension of these mechanisms is an essential step towards the definition of efficient flapping wing devices as there exists a tight connection between force production and leading edge vortex development. In particular, special attention should be paid to the vortex interactions that alter the leading edge vortex development, for example through the control of tip and trailing edge vortices.

\section{REFERENCES}

AONO, H., LiAnG, F. \& LiU, H. 2008 Near- and far-field aerodynamics in insect hovering flight: an integrated computational study. J. Expl Biol. 211, 239-257.

Birch, J. M. \& Dickinson, M. H. 2001 Spanwise flow and the attachment of the leading edge vortex on insect wings. Nature 412, 729-733.

Birch, J. M. \& Dickinson, M. H. 2003 The influence of wing-wake interactions on the production of aerodynamic forces in flapping flight. J. Expl Biol. 206, 2257-2272. 
Bos, F. M., Lentink, D., Van Oudheusden, B. W. \& Bijl, H. 2008 Influence of wing kinematics in hovering insect flight. J. Fluid Mech. 594, 341-368.

David, L., JARDin, T., Braud, P. \& FARCY, A. 2011 Time-resolved scanning tomography PIV measurements around a flapping wing. Exp. Fluids, doi:10.1007/s00348-011-1148-5.

DiCKINSON, M. H. \& GotZ, K. G. 1993 Unsteady aerodynamic performance of model wings at low Reynolds numbers. J. Expl Biol. 174, 45-64.

Dickinson, M. H., Lehmann, F. \& Sane, S. P. 1999 Wing rotation and the aerodynamic basis of insect flight. Science 284, 1954-1960.

Ellington, C. P. 1984 The aerodynamics of hovering insect flight. i.-v. Phil. Trans. R. Soc. Lond. B 305, 1-181.

Ellington, C. P., van den Berg, C., Willmott, A. \& Thomas, A. 1996 Leading-edge vortices in insect flight. Nature 384, 626-630.

JARDIN, T., DAVID, L. \& FARCY, A. 2009 Characterization of vortical structures and loads based on time-resolved PIV for asymmetric hovering flapping flight. Exp. Fluids 46, 847-857.

JeOng, J. \& Hussain, F. 1995 On the identification of a vortex. J. Fluid Mech. 285, 69-94.

KIM, D. \& GHARIB, M. 2010 Experimental study of three-dimensional vortex structures in translating and rotating plates. Exp. Fluids 49, 329-339.

LENTINK, D. \& DiCKInson, M. H. $2009 a$ Biofluiddynamic scaling of flapping, spinning and translating fins and wings. J. Expl Biol. 212, 2691-2704.

Lentink, D. \& Dickinson, M. H. 2009b Rotational accelerations stabilize leading edge vortices on revolving fly wings. J. Expl Biol. 212, 2705-2719.

MAXWORThy, T. 1979 Experiments on the Weis-Fogh mechanism of lift generation by insects in hovering flight. Part 1. Dynamics of the 'fling'. J. Fluid Mech. 93, 47-63.

Milano, M. \& GHARIB, M. 2005 Uncovering the physics of flapping flat plate with artificial evolution. J. Fluid Mech. 534, 403-409.

Poelma, C., Dickson, W. B. \& Dickinson, M. H. 2006 Time-resolved reconstruction of the full velocity field around a dynamically scaled flapping wing. Exp. Fluids 41, 213-225.

Ringuette, M. J., Milano, M. \& Gharib, M. 2007 Role of the tip vortex in the force generation of low-aspect-ratio normal flat plates. J. Fluid Mech. 581, 453-468.

SANE, S. P. 2003 The aerodynamics of insect flight. J. Expl Biol. 206, 4191-4208.

Shyy, W., Aono, H., Chimakurthi, S. K., Trizila, P., Kang, C. K., Cesnik, C. E. S. \& LIU, H. 2010 Recent progress in flapping wing aerodynamics and aeroelasticity. Prog. Aerosp. Sci. 46, 284-327.

ShyY, W., TrizIla, P., KAng, C. K. \& Aono, H. 2009 Can tip vortices enhance lift of a flapping wing? AIAA J. 47, 289-293.

SUN, M. \& LAN, S. L. 2004 A computational study of the aerodynamic forces and power requirements of dragonfly (Aeschna juncea) hovering. J. Expl Biol. 207, 1887-1901.

TAIRA, K. \& Colonius, T. 2009 Three-dimensional flows around low-aspect-ratio flat-plate wings at low Reynolds numbers. J. Fluid Mech. 623, 187-207.

WANG, Z. J. 2004 The role of drag in insect hovering. J. Expl Biol. 207, 4147-4155.

Williamson, C. H. K. 1996 Vortex dynamics in the cylinder wake. Annu. Rev. Fluid Mech. 28 477-539. 